\title{
Cerebral Vascular Hamartoma in a Campeiro Bulldog
}

\author{
Rafaela Magalhães Barros', Gustavo Andrade Gugelmin', Lucas Silva Barreto', Ana Maria de Souza Almeida', Tais \\ Meziara Wilson'2, Mizael Machado ${ }^{2}$, Alexandra Ariadine Bittencourt Gonçalves Pereira ${ }^{2}$ \& Márcio Botelho de Castro ${ }^{2}$
}

\begin{abstract}
Background: Vascular hamartomas $(\mathrm{VH})$ are rare or simply underdiagnosed injuries in veterinary medicine and represent a non-neoplastic developmental anomaly disorganization and proliferation of endothelial tissue. VH occur in any region of the body, however in the brain present clinical relevance related with the potential for spontaneous bleeding, adjacent tissue compression and convulsive activity. The aim of these report is to describe clinical, pathological and immunohistochemical features of a case of cerebrovascular hamartoma and highlight the diagnosis of these rare brain disorder in dogs.

Case: A 10-year-old male dog, a Campeiro Bulldog breed presented convulsions episodes and died before an elective surgical procedure for eyelid nodule removal. Three red nodules were observed in the brain, one between the parietal lobe and the left occipital lobe (in the medium suprasylviam sulcus), the other in the caudal region of the corpus callosum and the third one in the cerebellar cortex. Central nervous system, eyelids and most organs and tissues samples were collected, fixed in 10\% formaldehyde and processed for histopathological analysis. Histologically, in the eyelid was detected a sebaceous adenoma. The nervous system samples revealed well-differentiated sizes vascular structures with thin-walled and blood-filled, promoting compression of the brain. Normal neuropile was detected between the vascular structures substantiating cerebral vascular hamartoma diagnosis in the dog. Immunohistochemical assay was conducted with CD31 (monoclonal mouse antibody anti-CD31, Clone JC70A, Dako Corp.) and Von Willebrand factor (monoclonal mouse antibody anti-Von Willebrand factor, Clone F8/86, Dako Corp.) using the biotin-peroxidase-streptavidin method (PolyDetector Plus DAB-HRP, Bio SB) on CNS sections to confirm the vascular origin of the lining cells in the mass.

Discussion: Hamartomas are rarely reported in domestic animals and mostly are of vascular origin. $\mathrm{VH}$ in nervous tissues can trigger clinical signs related to hemorrhage, brain space occupation, compression and obstructive secondary hydrocephalus. In general, cerebral $\mathrm{VH}$ have a slow progression and usually affected animals are asymptomatic. On the other hand, cerebral vascular hamartomas may also cause clinical signs in very young animals about 15 to 16 months old. In humans, clinical presentation of VH may be related with acquired lesions such as trauma, ionizing radiation, and other central nervous system injuries. In the present case, the dog presented convulsion episode only at 10 years old during prechirurgical procedures. The morphological features of the vascular hamartoma we observed in the Campeiro bulldog is classified as capillary teleangiectasia composed by well differentiated capillaries lined with well differentiated endothelial cells interspaced by normal neuronal tissue. Histopathological and immunohistochemical assay are extremely important to differentiate hamartoma from other tumors, since they may be macroscopically similar. The immunolabeling of endotelial cells by anti-CD31 and anti-Von Willebrand factor antibodies in the cerebral VH, highlight the vascular origin of the masses detected. However, it is also important to perform systematic gross examination of the brain in detail to detect even the smallest injuries in asymptomatic animals avoiding underdiagnoses of cerebral VH.
\end{abstract}

Keywords: brain, tumor, malformation, hemorrhage, canine. 


\section{INTRODUCTION}

Vascular hamartomas ( $\mathrm{VH})$ are considered developmental anomalies, that represent an endothelial tissue disorganization and non-neoplastic proliferation in any region of the body $[1,5,8,9,20,22,23,25]$. $\mathrm{VH}$ may usually present low aggression to surrounding tissues depending on the location and also do not produce clinical signs, which lead to underdiagnoses [1]. The clinical importance of cerebrovascular hamartomas is related to the potential for spontaneous hemorrhage, compression of adjacent tissues and convulsive activity [20]. VH are rare in humans and animals and can occur isolated or in association with other injuries [1].

$\mathrm{VH}$ may be differentiated of hemangioma in nervous system due to the presence of normal neuronal tissue between the vessels, which is the main feature to the diagnosis of $\mathrm{VH}$ [4] and is absent or rare in cases of hemangiomas [17]. Immunohistochemical assay allow the identification of lining cells of vascular structures and also normal neural and glial components between the vessels in VH [20].

Generally, clinical diagnosis of cerebral VH is not considered in the diagnosis of brain disorders in dogs. Our report highlights the main pathological features relevant for identify cerebral $\mathrm{VH}$ and their differential diagnosis.

\section{CASE}

A 10-year-old male Campeiro Bulldog breed, weighing $35.5 \mathrm{~kg}$ received medical care for two years due to bilateral dry keratoconjunctivitis and develop a small nodule on the left eyelid. A surgical biopsy of the eyelid nodule had been scheduled, however, before the beginning of the preoperative examination, the patient presented convulsions episodes and died, and was taken for necropsy.

The main gross findings observed were multifocal reddish nodules ranging from $0.3-1.3 \mathrm{~cm}$ in diameter in the brain between the parietal lobe and left occipital lobe (suprasilvian sulcus medial region), cerebellar cortex and in the medial region of the telencephalon (specifically in the corpus callosum), promoting ventral compression of the lateral ventricle and parietal lobe (Figure 1). The lateral ventricles cavities were filled by red viscous fluid without apparent ventricular dilatation. In the left eyelid there was a small, circumscribed, whitish $0.4 \mathrm{~cm}$ nodule.
Central nervous system, eyelids and most organs and tissues samples were collected and fixed in $10 \%$ formalin, processed in paraffin, cleaved at 4 $\mu \mathrm{m}$ and stained with Hematoxylin and Eosin for histopathological analysis. In addition, immunostaining with CD31 (monoclonal mouse antibody anti-CD31, Clone JC70A) ${ }^{1}$ and Von Willebrand factor (monoclonal mouse antibody anti-Von Willebrand factor, Clone F8/86) ${ }^{1}$ was performed using the biotin-peroxidasestreptavidin method (PolyDetector Plus DAB-HRP) ${ }^{2}$ on CNS sections.

Microscopically, the nervous system samples revealed several vascular structures of varying sizes, lined by well-differentiated endothelial cells and filled with blood in the white and gray matter of the telencephalon, cerebellum and corpus callosum interspaced by areas of normal brain parenchyma (Figure 2). Endothelial cells lining the anomalous vascular structures showed strong immunostaining for CD31 (Figure 3) and von Willebrand factor. In the eyelid was detected a sebaceous adenoma composed by a proliferation of uniform cells organized in small lobes with central nucleus and condensed chromatin, and abundant foamy cytoplasm. No other gross or microscopic changes were detected in other tissues or organs. Clinical-pathological and immunohistochemical characteristics of lesions in the central nervous system allowed to conclude the diagnosis of cerebral vascular hamartoma.

\section{DISCUSSION}

Vascular hamartomas (VH) are non-neoplastic proliferations of vascular tissue. They are congenital defects of vascular formation and development, giving rise to normal tissue that is organized in a disorderly manner $[1,2,4,10,15,18,20]$ and are also considered benign tumors $[6,8,14,26]$. VH has been recently considered neoplastic because it exhibits chromosomal aberrations that are acquired through complete somatic mutations [11]. The link between abnormality and neoplasia is recognized due to disorganized overgrowth of vascular cells lining abnormal blood vessels and form a tumor-like mass [24]. In addition, the wide morphological variation of vascular hamartomas is considered a condition aiming to progress in a true neoplasia [17]

Hamartomas are rarely reported in domestic animals and mostly are of vascular origin [1,6,15,16,24], and frequently present no predisposition of age, breed or 


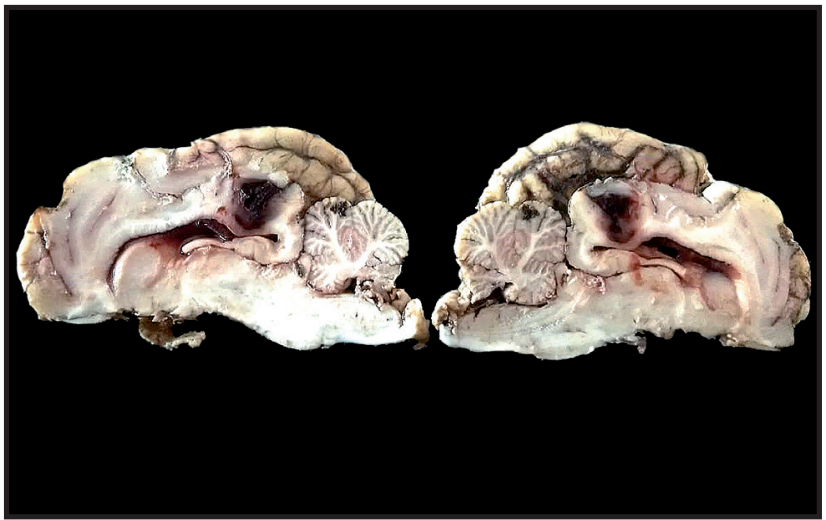

Figure 1. Brain, longitudinal section, dog. Multifocal reddish nodules in the parietal lobe, cerebellar cortex and corpus callosum.

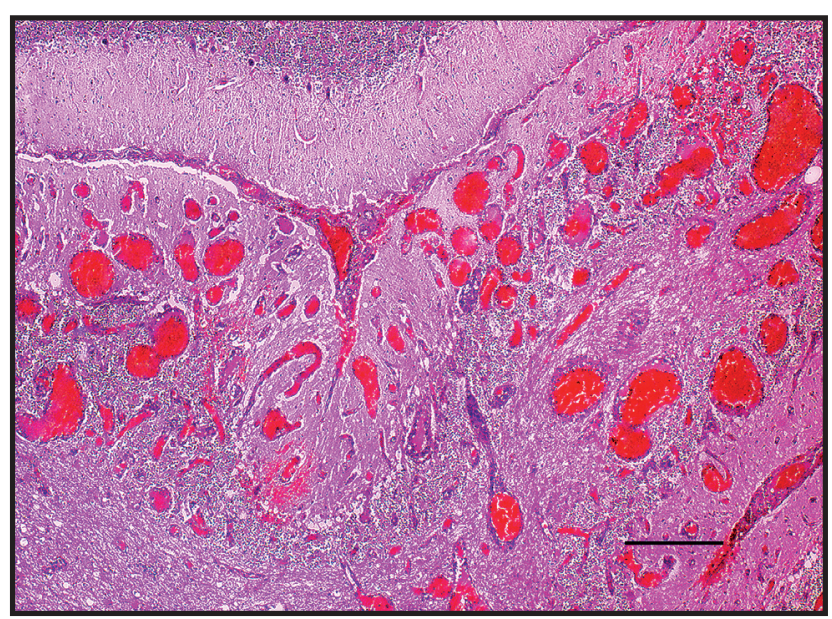

Figure 2. Cerebellum, dog. Vascular structures of varying sizesfilled with blood in the white and gray matter interspaced by areas of normal brain parenchyma $[\mathrm{H} \& \mathrm{E} ; \mathrm{Bar}=250 \mu \mathrm{m}]$.

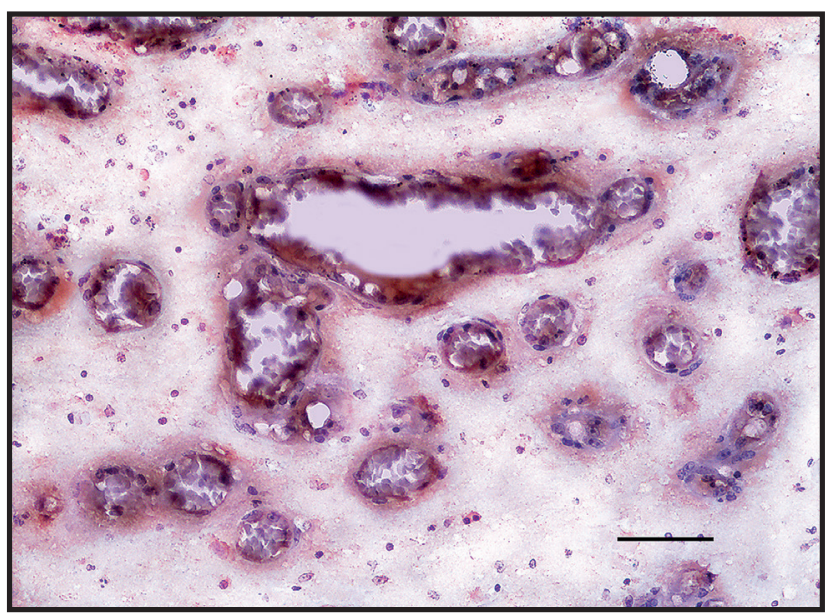

Figure 3. Telencephalon, dog. Endothelial cells lining the anomalous vascular structures presenting strong immunostaining for CD31 [Immunoperoxidase stain; $\operatorname{Bar}=50 \mu \mathrm{m}]$.

gender [20]. However, Scottish Terrier and Airedale Terrier dog breeds are highlighted to present a predisposition to develop VH [28]. Isolated cases of these vascular growths have been reported in the brain of dogs, cats, calves, goats and foals $[18,20,21]$. The telencephalon is considered the most frequent location of cerebral $\mathrm{VH}$ and mainly affect the piriform lobe [20]. Cerebral VH have been also reported in hippocampus [20] and cerebellum [21] of dogs and cats. In the Campeiro Bulldog, foci of VH were detected in the telencephalon, cerebellum and in corpus callosum. The multiple locations of $\mathrm{VH}$ in the central nervous system (CNS) of the dog observed here is uncommonly reported.

The clinical signs development observed in the dog could be attributed to the surrounding neural parenchyma compression $[4,16]$. Lesions progress for several years, but usually show no clinical signs and do not undergo malignant transformation, remaining unrecognized unless other circumstances arise, such as a significant increase in their size or pressure on other organs [1]. The up to 10 years old dog we are reporting here had never presented neurological signs. Some asymptomatic patients $[3,8,12,24]$ or with a delayed onset of clinical signs [13] were reported in animals and humans with cerebral VH. Despite the multifocal VH in the brain tissues of the dog, possibly the anomalous vascular growth did not cause significant injury in the surrounding tissues to produce neurological impairment. Vascular hamartomas have been diagnosed most frequently in symptomatic young patients [3,5,16,21,25,27], such as reported in cats with cerebellar and cervical cord vascular hamartomas $[16,21]$. The development of clinical signs is attributed to hemorrhage observed in and around the lesions, as well as its ability to act occupying space [7,13,16,20], which may lead to compression of the surrounding brain parenchyma and obstructive hydrocephalus secondary to the injury $[13,20]$. The Campeiro Bulldog presented convulsion episodes prior to death, probably in consequence of compression of neural tissues and hemorrhage to the ventricular cavity. Convulsions are the main clinical signs in patients with cerebral vascular hamartomas [18].

Cerebral VH in dogs are described as discrete softening multi-lobulated masses in the cerebral parenchyma with a red-brown color ranging from 0.4 to $2.0 \mathrm{~cm}$ in diameter [20]. This description is consistent with that identified in the dog of this report in different anatomical regions of the CNS. Hemorrhage and edema surrounding the mass [18], and vascular hamartomas in other organs $[13,21]$ were not detected in our case. VH have been also reported in the liver [5], ovary [2] and in the mandible involving practically the entire right incisor region [27] of domestic animals. 
The microscopic features observed in the CNS lesions of the dog support the diagnosis of cerebral $\mathrm{VH}$ $[4,13,20,21]$. Some reports of cerebral VH presented multiple thrombosis [5,18,20,21], varying degrees of hemorrhage and edema, neutrophil and macrophage infiltrate [18,20], mineralization and Purkinje cell chromalolysis [21], however, these pathological changes were not detected in the dog.

Important differential diagnoses for vascular hamartomas include, angiomatosis secondary to lymphedema and capillary hemangiomas [14,25], hemangioblastomas and these can be differentiated on the basis of histopathological features [28]. However, angiomatosis secondary to lymphedema are accompanied by characteristic hypoplasia of the deep lymphatic vessels and markedly dilated lymphatics, which were not seen in the current case. Capillary hemangiomas do not show neuropile present between vascular structures $[19,28]$. One of the main features of $\mathrm{VH}$ is that its component vessels are separated from each other by normal neural parenchyma $[9,14,19]$.

Vascular hamartomas are classified as arteriovenous, venous or cavernous malformations and capillary telangiectasia [13]. Arteriovenous malformations are considered the most common form in dogs [10]. The ce- rebral VH hamartoma observed in the Campeiro Bulldog presented vascular structures composed by small dilated capillary vessels interspaced by normal brain parenchyma, a morphological hallmark of capillary telangiectasias $[13,18]$. Additionally, the immunolabeling of endotelial cells by anti-CD31 and anti-Von Willebrand factor antibodies in the cerebral $\mathrm{VH}$, highlight the vascular origin of the masses detected [18].

Considering the similarities of the macroscopic aspect between $\mathrm{VH}$ and other vascular tumors, histopathological and immunohistochemical analysis are essential to the correct diagnosis of intra-cranial lesions. It is important to note, cerebral VH may be asymptomatic and usually an incidental finding at necropsy or slaughter. Therefore, clinical diagnosis of cerebral $\mathrm{VH}$ is generally not considered in the diagnosis of brain disorders in dogs. Our report highlights the main pathological features relevant for identify cerebral $\mathrm{VH}$ and their differential diagnosis.

\section{MANUFACTURERS}

${ }^{1}$ Dako North America Inc. Carpinteria, CA, USA.

${ }^{2}$ Bio SB. Santa Barbara, CA, USA.

Declaration of interest. The authors report no conflicts of interest. The authors alone are responsible for the content and writing of the paper.

\section{REFERENCES}

1 Bartyzel B.J., Max A., Gruszczynska J., Sobczak-Filipiak J.G.M., Mecik-Kronenberg T. \& Pankowski F. 2017. Hamartoma: a rare developmental disorder. Med Weter. 73(4): 202-207.

2 Benoit J.M., Lefebvre R.C., Mulon P.Y., Raggio I. \& Doré M. 2005. Ovarian vascular hamartoma in a cow. The Canadian Veterinary Journal. 46(11): 1026.

3 Booler H. 2008. Congenital Intrahepatic Vascular Anomaly in a Clinically Normal Laboratory Beagle. Toxicologic Pathology. 36(7): 981-984.

4 Borel N., Grest P., Junge H. \& Eser M.W. 2014. Vascular hamartoma in the central nervous system of a foal. Journal of Veterinary Diagnostic Investigation. 26(6): 805-809.

5 Braun U., Trosch L., Gerspach C., Brosinski K. \& Hilbe M. 2011. Ultrassonographic findings in a cow with vascular hamartoma of the liver: case report. BMC Veterinary Research. 7(1): 52.

6 Brisville A.C., Buczinski S., Chénier S. \& Francos D. 2012. A cardiac vascular hamartoma in a calf: ultrassonographic and pathologic images. Journal of Veterinary Cardiology. 14(2): 377-380.

7 Chambers B.A., Laksito M.A., Fliegner N.A., McCowan C., Beck C.\& Yates G.D. 2010. Nasal vascular hamartoma in a Domestic Shorthair cat. Australian Veterinary Journal. 88(3): 107-111.

8 Chanoit G., Mathews K.G., Keene B.W., Small M.T. \& Linder K. 2012. Surgical treatment of a pulmonary artery vascular hamartoma in a dog. Journal of the American Veterinary Medical Association. 240(7): 858-862.

9 Gualtieri M., Cocci A., Monti S. \& Olivero D. 2009. Surgical removal of a localized vascular hepatic hamartoma in a dog. Australian Veterinary Journal. 87(9): 360-362.

10 Ide T., Uchida K., Morozumi M. \& Nakayama H. 2009. Hamartoma in the Medulla blongata with Marked Mineral Deposits in a Dog. Journal of Veterinary Medical Science. 71(8): 1097-1100. 
11 Kumar V., Abbas A.K. \& Aster J.C. 2018. Neoplasia. In: Kumar V., Abbas A.K. \& Aster J.C. (Eds). Basic Pathology. 10th edn. Philadelphia: Elsevier, pp.189-242.

12 Lok C., Viseux V., Avril M.F., Richard M.A., Gondry-Jouet C., Deramond H., Desfossez-Tribout C., Courtade S., Delaunay N., Piette F., Legars D., Dreno B., Saiag P., Longy M., Lorette G., Laroche L. \& Caux F. 2005. Brain Magnetic Ressonance Imaging in Patients with Cowden Syndrome. Medicine. 84(2): 129-136.

13 Martin-Vaquero P., Moore S.A., Wolk K.E. \& Oglesbee M.J. 2011. Cerebral vascular hamartoma in a geriatric cat. Journal of Feline Medicine and Surgery. 13(4): 286-290.

14 Matos A.J.F., Duarte S., Lopes C., Lopes J.M. \& Gärtner F. 2007. Splenic hamartomas in a dog. Veterinary Record. 161(9): 308.

15 Nolf M., Maninchedda U., Belluco S., Lepage O. \&Cadoré J.L. 2014. Cecal vascular hamartoma causing recurrent colic in an Arabian mare. The Canadian Veterinary Journal. 55(6): 547.

16 Parkes J.D., Kline K.L., Riedesel E.A. \& Haynes J.S. 2009. A vascular hamartoma arising from the cervical spine of a cat. Journal of Feline Medicine and Surgery. 11(8): 624-627.

17 Saifzadeh S., Derakhshanfar A., Shokouhi F., Hashemi M. \&Mazaheri R. 2006. Vascular Hamartoma as the Cause of Hind Limb Lameness in a Horse. Journal of Veteterinary Medicine. 53(4): 202-204.

18 Sakurai M., Morita T., Kondo H., Uemura T., Haruna A. \& Shimada A. 2011. Cerebral Vascular Hamartoma with Trombosis in a Dog. Journal of Veterinary Medical Science. 73(10): 1367-1369.

19 Shirota M., Kawashima J., Nakamura T., Ogawa Y., Kamiie J. \& Shirota K. Vascular Hamartoma in the Uterus of a Female Sprague-Dawley Rat with an Episode of Vaginal Bleeding. Toxicologic Pathology. 41(7): 1011-1015.

20 Smith S.H. \& Van Winkle T. 2001. Cerebral Vascular Hamartomas in Five Dogs. Veterinary Pathology. 38(1): 108112.

21 Stalin C.E., Granger N. \& Jeffery N.D. 2008. Cerebellar hamartoma in a British Shorthair cat. Journal of Feline Medicine and Surgery. 10(2): 206-211.

22 Starost M.F. 2007. Solitary Biliary Hamartoma with Cholelithiasis in a Domestic Rabbit (Oryctolagus cuniculus). Veterinary Pathology. 44(1): 92-95.

23 Sugiyama A., Ozaki K., Takeuchi T. \&Narama I. 2007. Cardiac Vascular Hamartoma in Two Slaughtered Cattle. Journal of Comparative Pathology. 136(2-3): 202-205.

24 Takagi S., Kagawa Y., Hanazono K., Murakami S., Deguchi T., Izumi Y., Hosoya K., Kim S. \& Okumura M. 2018. Mandibular vascular hamartoma in a cat. The Journal of Veterinary Medical Science. 80(9): 1456-1458.

25 Taylor-Brown F.E., Lamb C.R., Martineau H., Muir C. \& Beltran E. 2018. Imaging Diagnosis - Imaging and Histopathologic Characteristics of a Vertebral Hamartoma in a Cat. Veterinary Radiology \& Ultrassound. 59(2): 12-16.

26 Tjostheim S.S., Kellihan H.T., Csomos R.A., McAnulty J. \& Steinberg H. 2015. Vascular hamartoma in the right ventricle of a dog: diagnosis and treatment. Journal of Veterinary Cardiology. 17(4): 321-328.

27 Tsuka T., Okamoto Y., Yamamoto N., Hayashi K., Morita T., Sunden Y., Murahata Y., Azuma K., Osaki T., Ito N. \& Imagawa T. 2018. Unilateral rostral mandibulectomy for gingival vascular hamartoma in two calves. Journal of Veterinary Science. 19(4): 582-584.

28 Yasuno K., Kobayashi R., Ohmuro T., Kamiie J., Sahara H. \& Shirota K. 2011. Caudal vascular hamartoma accompanied by aberrant arteriovenous structures in a dog. Journal of Veterinary Diagnostic Investigation. 23(5): 1051-1055. 